\title{
Praxis-Homepage
} nutzen das Inter-
net, also sollten
die Ärzte das
auch tun.

\section{Vielen Ärzten ist das Web egal - und warum das falsch ist}

\author{
Nur jede zweite Praxis hat eine eigene Website. Der Grund: Vielen \\ niedergelassenen Ärzten ist eine Homepage nicht wichtig. Ein Fehler, \\ sagt ein webaffiner Kollege - und erklärt, warum seiner Meinung nach \\ ein Internetauftritt für Praxischefs mittlerweile eigentlich Pflicht ist.
}

$\mathrm{N}$ ur etwa jede zweite Haus- und Facharztpraxis hat eine eigene Website - bei Gastronomiebetrieben sind es $89 \%$, bei Maklern 61 und bei Handwerkern $76 \%$. Diese Werte gehen aus einer repräsentativen Umfrage zu Mittelstand und Werbung im Auftrag des Telekommunikationsdienstleisters telegate hervor. $77 \%$ der Netz-Abstinenzler unter den Praxischefs begründen dies damit, eine Homepage sei für ihre Praxis nicht relevant. Ist die Web-Präsenz der Praxis für viele Ärzte ein zu komplexes Unterfangen? Diesen Eindruck vermittelt zumindest der Münchener Internist Dr. Christian Sailer im Gespräch. „Viele Praxen beginnen erst jetzt, sich mit dem Thema Internet richtig zu beschäftigen. Dabei ist es für die meisten aber immer noch zu komplex", meint Sailer.

\section{Aufschlussreich: der Blick aufs Geld}

Die Zurückhaltung manifestiert sich pekuniär: Die Hälfte der an der Studie teilnehmenden Ärzte investiert weniger als 1.000 Euro in die Praxis-Website, $20 \%$ stellen gar keine Mittel für den InternetAuftritt ihrer Praxis bereit.

Auch wenn es um den eigenen Auftritt bei Facebook, Xing oder Twitter geht, hält sich die Mehrheit der befragten Ärzte noch bedeckt. $80 \%$ gaben an, diese Medien seien für den Praxisbetrieb nicht von Belang. Das mag aber auch daran liegen, dass Ärzte der Schweigepflicht und strengen Datenschutzregeln unterliegen - und gerade bei den Social Media gibt es immer wieder Bedenken, ob sich diese Regeln hier einwandfrei einhalten lassen.

„Was die Sozialen Netzwerke angeht, kann ich diese Zurückhaltung sehr gut verstehen. Trend hin oder her, wichtig ist, was es der Praxis am Ende des Tages bringt, eine Fan-Page bei Facebook zu haben," so Sailer. Ein konkreter Nutzen sei für die Ärzte hier mehrheitlich noch nicht erkennbar.

\section{Suchmaschinenoptimierung ist das $\mathrm{A}$ und $\mathrm{O}$}

Nicht nachvollziehen kann Sailer, dass nur $8 \%$ der Praxen mit eigener Website eine Suchmaschinenoptimierung betreiben. „Ein Patient will als erstes wissen: Was macht der Arzt und wie ist der erste digitale Eindruck? Das geht nur über eine wirksame Website in Kombination mit sinnvoller Suchmaschinenoptimierung", so sein Plädoyer für die professionelle
Hervorhebung jeder Praxis-Website bei Google \& Co. Aus diesem Grund scheint es den Studienautoren nur logisch, dass $70 \%$ der ärztlichen Umfrageteilnehmer keine Erfolgskontrolle des (Online-)Praxismarketings durchführen. Sie legen ihren werblichen Investitionsfokus auf bewährte Formate außerhalb des Internets.

\section{Neue Patienten, Stärkung} im Wettbewerb

„Viele niedergelassene Ärzte trauen sich einfach noch nicht", deutet Sailer diese Studienergebnisse. Seiner Ansicht nach ist eine Internet-Präsenz sowohl für Ärzte in ländlichen Gebieten als auch in Ballungszentren Pflicht und keine Kür, da sie vor allem dafür sorge, von neuen Patienten gefunden zu werden und sich im Wettbewerb von anderen Facharztpraxen zu unterscheiden.

In Städten mit hoher Arztdichte trage gerade die Suchmaschinenoptimierung für spezielle Keywords dazu bei, dass potenzielle Patienten schnell die medizinischen Schwerpunkte und Spezialisierungen der jeweiligen Praxis fänden. „Dafür fahren sie dann auch schon mal quer durch die ganze Stadt", so Sailer.

Matthias Wallenfels 